\title{
ATTITUDES AND OPINIONS OF YOUNG POPULATION ON THE CONCEPT OF THE INTERNET AS A FORM OF INTEGRATED MARKETING COMMUNICATION
}

Sanja Bijakšićl, Brano Markićl, Arnela Bevanda

University of Mostar, Mostar, Bosnia and Herzegowina

\section{ABSTRACT}

Integrated Marketing Communication is both the system and the process of development and implementation of multiple forms of communication with the market. Its long-term goa is to strengthen relations with the current customers but also to attract new customers. Therefore, the primary goal of integrated marketing communication is to have influence on the behaviour of customers in order to shape and change their views and opinions. The marketing communication mix consists of advertising, direct marketing communications, sales promotion, personal selling, public relations, publicity and outdoor advertising. This study analyzes the attitudes and opinions of young population regarding the Internet as one of the most important media market communications. The instrument of data collection was a questionnaire and hypotheses were tested by using the chi-square test and confirmatory factor analysis.

\section{JEL CLASSIFICATION \& KEYWORDS}

M31 INTEGRATED MARKETING COMMUNICATION THE INTERNET — HI-SQUARE TEST — CONFIRMATORY FACTOR ANALYSIS

\section{INTRODUCTION}

Bijakšić at al (2013) writes the Internet is not only a contemporary medium, but it represents a new paradigm of marketing by putting the consumer in the role of an offer creator, the most active member of controlling the entire process of production and sales. The impact of the Internet cannot be predicted, because technologica improvements cannot be predicted, but on the basis of the existing trends, it is clear that the Internet is the main

of medium for advertisers who want to be noticed and successful.

The analysis of attitudes, opinions and behaviour of the Internet users regarding their socio-economic characteristics, and the main activities and objectives of the use of its services are an important source of data for marketing and management in managing and directing the organizational system to goal-setting.

Bijakšić at al (2014) states that the concept of attitude is often equated with the other concepts such as: opinion, view on the world. However, its essence in marketing is different. Attitude is primarily an emotional experience unlike understanding that is oriented and based on intellect. Opinion is a personal statement about the position, event object or situation. There are several factors that affect shaping the attitude. Some are linked to the personality of user services, experiences, information, psychosocia interactions. Client behaviour is determined by their attitudes towards the object, destination, brand. Naturally, in the same market, the same views of customers and consumers can be different at different times.

\footnotetext{
'sanja.bijaksic@sve-mo.ba

" branom@sve-mo.ba

www.journals.cz
}

It is assumed in the study that is possible to understand the key factor that determines the reasons for the use of the Internet by young population. The young generation of university students use the Internet on the everyday basis, where some computer services provide better awareness on the events, people and processes in the world, some of them are primarily taught by accessing databases from the narrow professional areas, while others improve communication with other people and so on. According to this research there are three main hypotheses set up:

$\mathrm{H} 1$ : There are differences in the time spent on the Internet due to sex of service users.

$\mathrm{H} 2$ : There are differences in the objectives that the Internet service users want to achieve by their use due to sex of service users.

H3: The main factor of using the Internet is to improve communication with the environment and it dominates compared to other factors.

\section{Integrated marketing communication and the Internet}

The Internet is currently the most perspective form of interactive media. Radio, television, film, various magazines, news, countless shop-windows, posters, the Internet, all of these factors affect the psyche of a modern man. However, one does not react to everything, but only on the issues that fit his current needs. The Internet is a means of publicity, advertising space, a meeting point of supply and demand, economic space. Through the web sites many companies transmit information about themselves on social networking sites and interact with audiences. Therefore, the Internet nowadays is not only the technological infrastructure, but also the global economic area that encourages competition, new market and organizational structure, but also allows companies to supply determining variable (different) price for different customers.

Bijakšić at al (2014) denotes Internet is an external determinant of pricing technology nature that enables a company to define flexible pricing strategies of products or services. The Internet also holds an enormous potential to change pricing strategies. A key advantage offered by the Internet to determine the price is transparency.

The idea of transparency is based on the possibility of rapid and detailed searching and browsing the competitive prices of products and services offered online. The customer can get almost perfect information about the prices of the same or similar products from a large number of bidders. This fact affords attributes to the Internet as an efficient market. It changes the pricing strategies at the same time enabling the bidder to determine fixed prices for all customers and variable rates for different customers but also bartering.

Customers have access to the specific applications of Shopbots (consumer comparison with the price lists, traderobots for purchase), and they allow the customers continuous obtaining of information on prices and sellers. 
This fact raises questions of consumer behaviour, but also the impact of the Internet and applications for monitoring of prices of the same or similar products on the policy and strategy of price vendor.

The Internet is currently the fastest growing marketing channel. The service is distributed in the provision of services, thus marketing channel services are much shorter. It is the application of information technologies and the Internet that enabled new forms of commerce (e- commerce) as well as promotion (advertising on the Internet). The modern consumer is seeking a new experience when purchasing and often has less time for shopping.

The architecture of the Internet, the way of addressing a computer, parcel transfer mode or safety aspects of the Internet are not in the focus of the interest of marketing experts. It is interesting only as a medium of integrated marketing communications, which affects the behaviour of consumers, facilitates and increases the transmission and presentation of the desired information to a target audience enhancing the synergic efficacy that drives the customer to action and makes it easier to build long-term relations with customers.

The Internet opens up the application of the new integration at all levels. Due to widespread usability and easy access, the Internet allows new business activities, social interaction and new forms of communication with the target market.

Kesic (2003) agrees that one of the more detailed definitions of integrated marketing communications gave the American communicator D.E.Shulz [4].

"Integrated Marketing Communication is the process of development and implementation of various forms of persuasive communication with customers and potential customers at a given time. Its aim is to influence or directly target the behaviour of customers and uses all appropriate forms of communication that may affect the behaviour of customers. The Internet is a communication medium that substitutes the printed media but also the verbal with interactive communication. It is therefore interesting from the perspective of integrated marketing communication to investigate whether there is a difference of the Internet users due to their sex, in the objectives of the Internet use, the time spent on the Internet during the day and the key factors that motivate people to use its services. Nowadays, there is no a company that has not involved the Internet in the marketing communication system but the insufficient use of its potential has also been pointed out.

The questions in the questionnaire, which reveal the behaviour of younger people related to using the Internet services include the number of hours of free time during the day, the media, which often follow, the frequency of access to the Internet services, the time spent on the Internet during the day, use of the Internet in the process of purchasing products, while opinions on the Internet as a form of integrated marketing communications detect reasons for using the Internet, the feeling of isolation from friends and family due to the daily use of the Internet, leaving the Internet as a form of communication, its weaknesses, its impact on business, friendship, family, etc.

A detailed list of questions in the questionnaire and the results of the tested hypotheses are given in the survey results.

\section{Results of research}

The first step in data analysis and hypothesis testing is to load data from a questionnaire in the $\mathrm{R}$ language by using

www.journals.cz the tool (read the table). The data group contains the answers of twenty-one respondents. Considering the variables, 18 out of 21 are related to the attitudes and opinions of students on the Internet as a medium in the system of integrated marketing communications. These questions describe the use of the Internet by young student population of undergraduate and graduate studies. A structured questionnaire contains the following questions and answers ${ }^{1}$.

1. The main reason for using the Internet is: $1=$ learning, $2=$ awareness enhancement, $3=$ to have fun, $4=$ to communicate, 5 = purchase, $6=$ something else $-(R u)$.

2. Does the Internet positively affect your sociability? $(0=$ no, $1=$ yes, $2=$ I do not know $)-(P d)$.

3. The Internet use increases your isolation from friends and family $(0=$ no, $1=$ yes $)-(Z i)$.

4. Do you think you could be without the Internet services? $(1$ = yes, 2 = temporarily, $3=\mathrm{no})-\mathrm{Mi}$

5. Internet can be harmful to young people because it shortens their time to spend with family and friends (Likert scale 1-5) - MS.

6. Communication over the Internet cannot replace direct communication with the person (Likert scale 1-5) - ZK.

7. Internet increases the power of friendship (Likert scale 1-5) - Spr.

8. Internet is important to communicate with consumers (Likert scale 1-5) - Kp.

9. Internet influences the public opinion (Likert scale 1-5) $\mathrm{Jm}$.

10. Internet affects work and leisure (Likert scale 1-5) - Psv. 11. Internet is changing habits (Likert scale 1-5) - Nm.

12. Do you use the Internet in the process of purchasing products $(0=$ no, $1=y e s)-$, CPR.

13. Internet is crucial to communicate with people of the same professional orientation (Likert scale 1-5) - Po.

In the analysis of data and testing the hypotheses, $\mathrm{R}$ programming language will be used with its packages and functions.

First, loading the data from the questionnaire (respondents' answers) from the file "C: //rSaHdzu.csv" stored in the root directory of the hard disk. It provides the following command of $R$ language:

>emPC<-read.table("C://rSaHdzu.csv",header=T,sep=";")

$>\mathrm{EmPC}$

\begin{tabular}{|rrrrrrrrrrrrrrrrrrrrrrr|r|r|}
\hline \multicolumn{11}{|c|}{ Sp Gs Sv Me Vm Pi Vp Vi Ru Pd Zi Mi Ms Zk Spr Kp Jm Psv Nm Kpr Po } \\
\hline 1 & 1 & 3 & 2 & 4 & 4 & 1 & 1 & 2 & 4 & 1 & 0 & 2 & 3 & 3 & 5 & 5 & 5 & 4 & 4 & 1 & 5 \\
2 & 1 & 3 & 3 & 4 & 4 & 1 & 0 & 1 & 3 & 1 & 1 & 2 & 3 & 3 & 4 & 5 & 5 & 5 & 2 & 1 & 4 \\
\hdashline & 0 & 0 & 4 & 3 & 4 & 4 & 1 & 1 & 4 & 1 & 1 & 0 & 3 & 4 & 3 & 3 & 4 & 4 & 3 & 2 & 1 & 5 \\
\hline
\end{tabular}

The first step in the data analysis is to test the internal consistency of the questions in the questionnaire by using Cronbach alpha coefficient of reliability. In $\mathrm{R}$ language is sufficient to load the psych package and then enter the function alpha ():

1 There are possible answers in the brackets and the way of encoding their numerical values. Below is the abbreviation used for the questions in the data analysis. For example, the first question is the questions in the data analysis. For example, the first question is
regarding gender. Possible answers of respondents are male or female, where the value characteristics for males are coded with numerical value of 0 and for females with numerical value of 1 . Feature name regarding sex is named $\mathrm{Sp}$ in data analysis. 


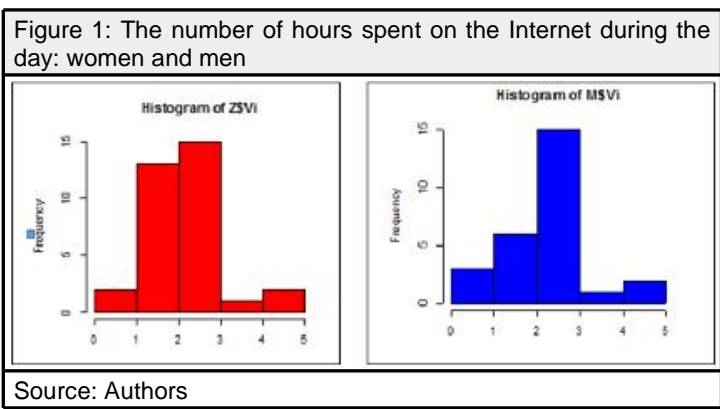

$>$ library (psych)

$>$ alpha (EMPCO)

reliability analysis

Call: alpha $(x=$ EMPCO)

raw_alpha std.alpha G6 (smc) average_r S / N ase mean sd

$0.530 .570 .740 .061 .30,0932.20 .25$

lower alpha upper $95 \%$ confidence boundaries

$0: 350: 530.72$

The value of coefficient alpha is 0.53 which can be accepted as the confidence interval (Cronbach alpha $<0.5$ is unacceptable).

The first hypothesis of the study $(\mathrm{H} 1)$ : There is no difference between young people in terms of time spent on the Internet regarding their gender.

This hypothesis will be verified by a simple descriptive statistics and visualization of data by using a histogram and then the chi-square test.

Sort out the of male and female population in the sample, and then by using histogram display the responses of frequency of time spent on the Internet during the day, regarding female and male young adults. $>Z$ subset (EMPCO, Sp = = 1)

$>$ hist $(Z \$$ are, $c o l=$ "red" $x l a b=$ "The number of hours spent on the Internet during the day - women")

$>$ hist $(\mathrm{M} \$$ are, col = "blue", xlab = "The number of hours spent on the Internet during the day - men")

The histogram shows that the largest number of women and men daily spend between three to four hours on the Internet. Distinctions in the gender gap in the terms of distribution of time spent on the Internet during the day are very small. However, safe testing of $\mathrm{H} 1$ hypothesis provides Chi-square test. Therefore, the use of chi-square test will examine the existence of differences between women and men in the terms of time spent on the Internet.

First, calculate the appropriate frequency of the time spent on the Internet during the day regarding men and women. It is sufficient to write the commands:

$>=$ hk table (EMPCO \$ Sp, EMPCO \$ Vi)

$>\mathrm{hk}$

Tabulation of the results can be seen in the table below:

\begin{tabular}{|c|c|c|c|c|c|c|}
\hline Gender & up to $30 \mathrm{~min}$ & $\begin{array}{l}\text { up to } \\
\text { hour }\end{array}$ & an $\begin{array}{l}\text { up to } \\
\text { hours }\end{array}$ & \begin{tabular}{|l|l|} 
two & $\begin{array}{l}\text { between } \\
\text { and } 4 \text { hours }\end{array}$ \\
\end{tabular} & $\begin{array}{l}\text { between } 5 \\
\text { and } 7 \text { hours }\end{array}$ & $\begin{array}{l}5 \text { more than } 7 \\
\text { s hours }\end{array}$ \\
\hline Male & 1 & 2 & 6 & 15 & 1 & 2 \\
\hline Female & 1 & 1 & 13 & 15 & 1 & 2 \\
\hline
\end{tabular}

www.journals.cz
After calculating the appropriate frequency, it identifies the function:

$>$ Chisq.test (hk)

Pearson's chi-squared test

data: $\mathrm{HKX}$-squared $=2.3356, \mathrm{df}=5, \mathrm{p}$-value $=0.801$

Since the $p$-value of 0.801 is greater than 0.05 on significance level, the null hypothesis cannot be rejected regarding the time spent on the Internet, regardless of gender.

The following hypothesis in the study $(\mathrm{H} 2)$ : there is no difference regarding the gender in terms of reasons for using the Internet.

Firstly, histograms will show the relevant distribution of frequencies and visually perform the validity of the hypothesis. The conclusion will be verified afterwards by applying Chi-square test. It is certainly interesting to examine and identify the most common reasons for using the Internet. Frequency reasons of the Internet use by young population (the students' population) is shown in Figure 2. (by using a histogram) ${ }^{2}$.

$>$ hist $($ EMPCO \$ Ru, col = "pink", xlab = "Reasons (activities) for using the Internet")

Figure 2: The frequency reasons for using the Internet

Histogram of emPC\$Ru

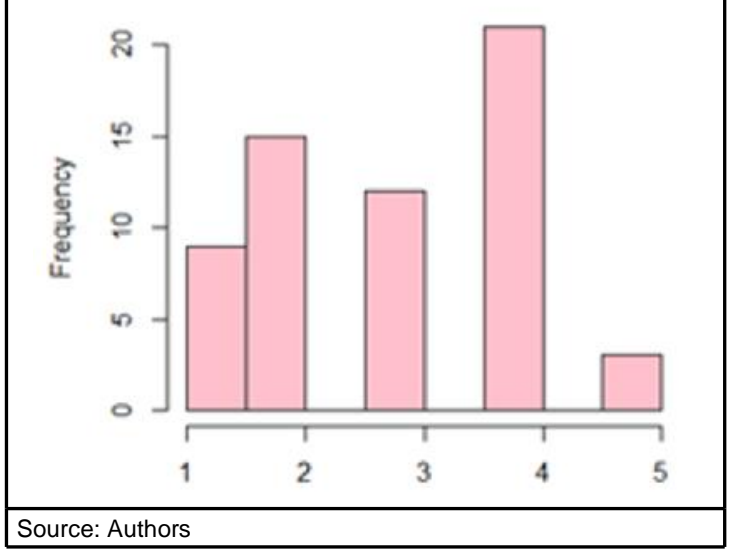

The histogram shows that the three most common reasons for using the Internet are: communication, information, entertainment and learning. It is interesting to re-analyze the existence of differences between women and men. In fact, the visualization in the form of histogram will immediately provide full information by using the following commands of $\mathrm{R}$ language:

$>$ hist (Z \$ Ru, col = "red" xlab = "Reasons (activities) for using the Internet - Women")

$>$ hist (M \$ Net, col = "blue", xlab = "Reasons (activities) for using the Internet - Men").

Histograms show that the similarities between women and men while using the Internet are great once again. Both sexes mostly use the Internet for communication. However,

2 Histogram displays the value of time spent during the day, which is coded as follows:

0 - values up to $30 \mathrm{~min} ; 1$ - up to an hour; 2 - up to two hours; 3 - between three and four hours; 4 : between five and seven hours; 5 - more than seven hours 


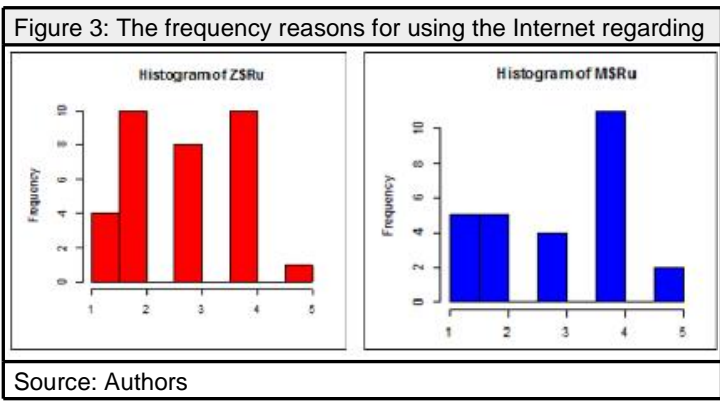

it is interesting that apart from communication, female population often frequently uses the Internet services in order to be informed on a variety of contents and entertainment. Younger male population uses the Internet services for learning more often than female population.

However, visualization is not enough for the general conclusions, nor can it verify the adjusted hypothesis with an adequate level of significance. Therefore, it is necessary to apply the chi-square test, in order to calculate the frequency of activities of people on the Internet, in examining the function regarding their gender.

$>=$ al table (EMPCO \$ Sp, EMPCO \$ Ru)

$>$ al

\begin{tabular}{|c|c|c|c|c|c|}
\hline Gender & Learning & \begin{tabular}{|l|} 
Getting \\
information
\end{tabular} & Entertainment & t Communication & Purchase \\
\hline Male & 5 & 5 & 4 & 11 & 2 \\
\hline Female & 4 & 10 & 8 & 10 & 1 \\
\hline
\end{tabular}

Source: Authors

After calculating the appropriate frequency, it activates the function of $R$ language:

$>$ Chisq.test (Al)

Pearson's chi-squared test

data: Al

X-squared $=2.9213, \mathrm{df}=4, \mathrm{p}$-value $=0.5711$.

Since the $p$-value ( $p$-value) of 0.5711 is greater than 0.05 of the assumed significance level, the null hypothesis cannot be rejected, in which the activities and methods of using the services on a global computer network are independent regardless of the users' gender.

The following hypothesis $(\mathrm{H} 3)$ is: the main factor of the Internet use is to improve communication with the environment and that this factor dominates in relation to other factors.

This hypothesis is examined by using multivariate analysis methods. It is possible to apply a confirmatory factor analysis and principal component analysis.

With the help of confirmatory factor analysis, three factors will be extracted . Factors are determined by the answers, which are numbered from 13 to 19 in the questionnaire. They are extracted in the data frame by the following command of $R$ language:

$>\mathrm{fA}=$ as.data.frame (EMPCO [13: 19], drop $=$ FALSE)

The three most important factors are determined with the command:

$>$ fit $<-$ factanal $(\mathrm{fA}, 3$, rotation $=$ "varimax")

$>$ fit

www.journals.cz
Call:

Factanal $(\mathrm{x}=\mathrm{fA}$, factors $=3$, rotation $=$ "varimax")

Uniquenesses:

\begin{tabular}{|l|l|l|l|l|l|l|}
\hline Ms & Zk & Spr & Kp & Jm & Psv & Nm \\
\hline 0.005 & 0.005 & 0.762 & 0.005 & 0.723 & 0.82 & 0.975 \\
\hline
\end{tabular}

Loadings:

\begin{tabular}{|l|c|c|c|}
\hline & Factor 1 & Factor 2 & Factor 3 \\
\hline Ms & 0.996 & & \\
\hline Kk & & & 0.997 \\
\hline Spr & 0.335 & & -0.347 \\
\hline $\mathrm{Kp}$ & & 0.997 & \\
\hline $\mathrm{Jm}$ & -0.179 & 0.49 & \\
\hline $\mathrm{Psv}$ & -0.401 & 0.127 & \\
\hline $\mathrm{Nm}$ & & -0.14 & \\
\hline
\end{tabular}

\begin{tabular}{|l|c|c|c|}
\hline & Factor 1 & Factor 2 & Factor 3 \\
\hline SS loadings & 1.298 & 1.279 & 1.129 \\
\hline Proportion Var & 0.185 & 0.183 & 0.161 \\
\hline Cumulative Var & 0.185 & 0.368 & 0.529 \\
\hline
\end{tabular}

The test of the hypothesis shows that three factors are sufficient. The chi square statistic is 5:46 on 3 degrees of freedom. The $p$-value is 0.141 .

P-value of 0.141 . is again higher than 0.05 and the null hypothesis cannot be rejected that the three factors are sufficient.

The first factor emphasizes the weakness of the Internet because it can significantly reduce the time that young people might spend with their families and friends. Another factor is the communicative power of the Internet, which is manifested under the influence of shaping the public opinion $(\mathrm{Jm})$ and the importance of communication with customers (consumers). Another factor directly emphasizes marketing dimension of the internet and its importance in shaping public relations.

The following commands provide a graphical display of the relation between factor 1 and factor 2 (weaknesses of the Internet and its communicative power) $)^{3}$ :

$>$ print (fit, digits $=2$, the cutoff $=.3$, sort $=$ TRUE)

$>$ load <- fit \$ loadings [1: 2]

$>$ plot (load, xlab = "The weaknesses of the Internet," ylab = "Communicative power of the Internet", type = "n")

$>$ text $($ load, labels $=$ names $(\mathrm{fA}), \mathrm{cex}=.8, \mathrm{col}=$ "blue", pch $=21$

The third factor is the indifference of the Internet towards interpersonal communication. The Internet services cannot reach the strength and importance of interpersonal communication.

Factor analysis confirms the $\mathrm{H} 3$ hypothesis, that the main power of the Internet, which attracts young population is its communicative power with the environment and that it is one of the most important factors of the Internet use. In addition, the Internet shows its weaknesses in terms of neglecting interpersonal communication with family and friends (the first factor) and it cannot replace the importance of direct interpersonal communication (the third factor).

${ }^{3}$ Varimax rotation factor is used. 


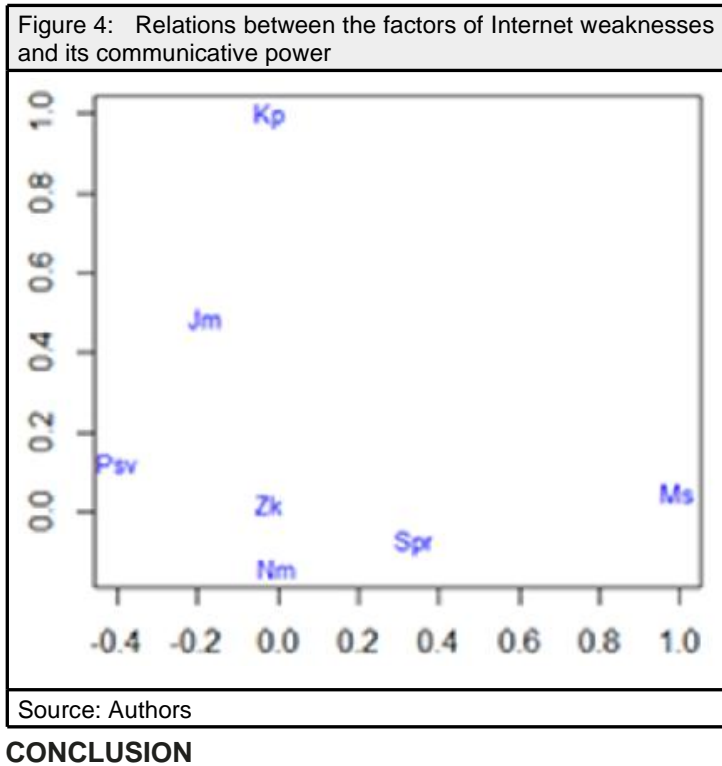

CONCLUSION

Integrated marketing communication is a process used to influence consumer behaviour. The Internet is an important medium of integrated marketing communication which is used to simplify and accelerate the transmission and presentation of the desired data to a target audience, it simplifies the process of building long-term relations with the customers, but it can also trigger the action of a purchase. The Internet opens up a possibility for new business activities, social interaction and new forms of communication with the target market. This study analyzes the attitudes and opinions of graduate and undergraduate students on the Internet as a medium of integrated marketing communication. Results of the study shows that there is neither difference in terms of time spent during the day on the Internet between male and female population nor the significant differences in the predominant activity and the reasons for their use. Applied confirmatory factor analysis shows that the dominant factor in using the Internet is its power of communication with the environment which is particularly important for positioning the Internet as a form of integrated marketing communication comparing to other forms of communication. The marketing strategy of building an integrated marketing communication must take into account dominance of the Internet as a medium of communication with customers with relevant background. Hypothesis testing has proved satisfactory implementation of power and simplicity of $R$ language.

\section{REFERENCES}

Bijakšić, S., S. Bevanda, Markić B., (2014) Marketing and Metrics, HKDU, Head Branch Mostar.

Bijakšić, S., B. Markić, Bevanda A., (2013) Text Mininig and analysis of attitudes in relation to social networks, Sibenik.

Kesic, T., (2003) Integrated Marketing Communications, Opinio, Zagreb. 